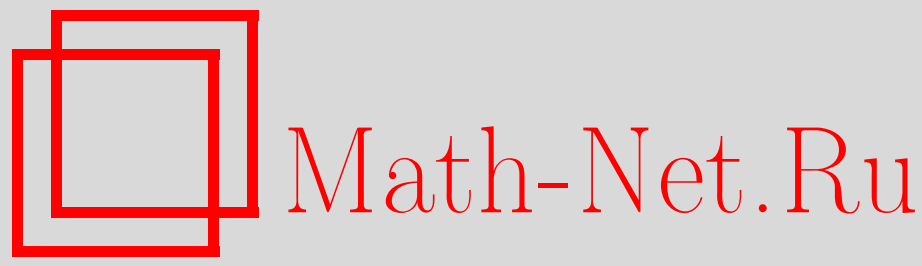

И. Я. Арефьева, И. В. Волович, Е. В. Писковский, Скатывание в модели Хиггса и эллиптические функции, TMФ, 2012, том 172, номер 1, 138-154

DOI: https://doi.org/10.4213/tmf6925

Использование Общероссийского математического портала Math-Net.Ru подразумевает, что вы прочитали и согласны с пользовательским соглашением http://www.mathnet.ru/rus/agreement

Параметры загрузки:

IP: 3.85 .7 .115

26 апреля 2023 г., 03:48:31

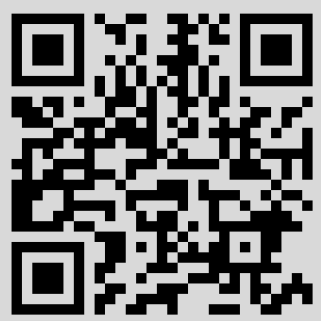




\title{
ФИЗИКА
}

Том 172, № 1

июль, 2012

(C) 2012 г. И. Я. Арефьева*, И. В. Волович*, Е. В. Писковский*

\section{СКАТЫВАНИЕ В МОДЕЛИ ХИГГСА И ЭЛЛИПТИЧЕСКИЕ ФУНКЦИИ}

\begin{abstract}
Асимптотические методы в нелинейной динамике, например метод усреднения Боголюбова-Крылова и теория Колмогорова-Арнольда-Мозера, используются обычно для улучшения теории возмущений в режиме малых колебаний. Однако в ряде задач нелинейной динамики, в частности для уравнения Хиггса в теории поля, представляет интерес не только режим малых колебаний, но и режим скатывания. В космологии Фридмана важны режимы как медленного, так и быстрого скатывания. Предлагается асимптотический метод решения уравнения Хиггса в режиме скатывания. Показано, что для улучшения теории возмущений в режиме скатывания эффективным оказывается разложение известного решения в терминах эллиптических функций не по тригонометрическим функциям, как в методе усреднения в режиме малых колебаний, а по гиперболическим функциям. Приводится набросок оценки точности второго приближения. Рассмотрено также уравнение Хиггса с затуханием.
\end{abstract}

Ключевые слова: асимптотические методы в нелинейной динамике, скатывание, модель Хиггса.

\section{1. ВВЕДЕНИЕ}

Известные асимптотические методы в нелинейной динамике такие, как метод усреднения Боголюбова-Крылова, метод медленно изменяющихся параметров Ляпунова, методы Пуанкаре, Ван-дер-Поля и теория Колмогорова-Арнольда-Мозера, используются обычно для улучшения теории возмущений в режиме малых колебаний [1]-[4]. Даже сам термин "нелинейная механика" нередко используется как синоним теории нелинейных колебаний [1].

Однако для уравнения Хиггса в теории поля [5], уравнения Фридмана в космологии [6], [7] и в ряде других задач нелинейной динамики представляет значительный интерес не только режим малых колебаний, но и режим скатывания (см., например, работы [8], [9] и приведенные в них ссылки). В режиме малых колебаний рассматривают возмущения условно периодических движений, в частности в окрестности решений линейного уравнения гармонического осциллятора

$$
\ddot{q}+\omega^{2} q=0, \quad \omega>0,
$$

*Математический институт им. В. А. Стеклова РАН, Москва, Россия. E-mail: arefeva@mi.ras.ru 
где $q=q(t)$ - вещественнозначная функция времени $t$. В режиме скатывания (или подъема) рассматриваются возмущения движения в окрестности решений следующего линейного уравнения:

$$
\ddot{q}-\mu^{2} q=0, \quad \mu>0 .
$$

В настоящей работе мы предлагаем развитие метода теории возмущений гамильтоновой системы в режиме скатывания, которое заключается в построении приближенных решений с использованием разложения по гиперболическим функциям, и рассматриваем этот метод на примере важного модельного уравнения Хиггса, в том числе с затуханием. Установлена интересная аналогия полученных результатов с формулами метода усреднения. Уравнения (1.1) и (1.2) связаны заменой $\omega=i \mu$, однако для доказательства оценки приближения в режиме скатывания метод усреднения непосредственно неприменим, и мы используем другой метод - разложение эллиптических функций по гиперболическим ${ }^{1)}$. Приведен набросок оценки точности второго приближения.

Для уравнения ангармонического осциллятора (уравнения Дюффинга) известно решение в терминах эллиптических функций, причем формулы метода усреднения для малых колебаний соответствуют разложению эллиптической функции в ряд Фурье по тригонометрическим функциям. В настоящей работе рассматривается уравнение Хиггса, и с использованием известного решения в терминах эллиптических функций исследован режим скатывания. Рассмотрено также уравнение Хиггса с затуханием. Показано, что для улучшения теории возмущений в режиме скатывания эффективным оказывается разложение не по тригонометрическим функциям, как в режиме осцилляций, а по гиперболическим функциям.

Хорошо известно, что простейшие уравнения нелинейной динамики допускают точное решение, которое можно получить, используя эллиптические функции. Соответствие между этими точными решениями и асимптотическим методом усреднения рассмотрено в книге [2], где исследуется уравнение ангармонического осциллятора с вещественной частотой,

$$
\ddot{q}(t)+\omega^{2} q(t)=-\epsilon q^{3}(t), \quad \omega>0, \quad \epsilon>0,
$$

а также проведено численное сравнение приближенного и точного решений. Применение приближенных решений в функциональной механике можно найти в рабо$\operatorname{Tax}[10],[11]$.

В настоящей работе в отличие от книги [2] мы рассматриваем уравнение ангармонического осциллятора с мнимой частотой (уравнение Хиггса):

$$
\ddot{q}(t)-\mu^{2} q(t)=-\epsilon q^{3}(t), \quad \mu>0, \quad \epsilon>0 .
$$

$\mathrm{C}$ помощью представления решения в терминах эллиптических функций мы исследуем режим скатывания.

Как известно, при помощи сдвига на константу $q(t)=x(t)+\mu / \sqrt{\epsilon}$ уравнение (1.4) переводится в уравнение для нелинейных осцилляций

$$
\ddot{x}+2 \mu^{2} x=-\epsilon x^{3}-3 \sqrt{\epsilon} \mu x^{2},
$$

1) Было бы интересно рассмотреть возможное соответствие метода разложения по гиперболическим функциям, используемого в настоящей работе при не слишком больших значениях времени $t$, с предложенным в работе [4] развитием первого метода Ляпунова, который эффективен при $t \rightarrow \infty$. 
однако это преобразование не позволяет исследовать режим скатывания более эффективно, чем при непосредственном рассмотрении исходного уравнения Хиггса.

В разделе 2 мы обсуждаем гиперболический аналог метода усреднения. В разделе 3 приведены в удобной для нас форме известные решения уравнения Хиггса в терминах эллиптических функций. Разложения эллиптических функций по гиперболическим с соответствующими оценками рассмотрены в разделе 4. Наконец, набросок оценки второго приближения представлен в разделе 5 .

\section{2. ГИПЕРБОЛИЧЕСКИЙ АНАЛОГ МЕТОДА УСРЕДНЕНИЯ}

Простейшее одномерное уравнение колебаний в нелинейной динамике имеет вид

$$
\ddot{q}+\omega^{2} q=\epsilon f(q, \dot{q})
$$

где $q=q(t)$ - вещественнозначная функция времени, частота $\omega$ - положительное число, $\epsilon$ - малый параметр. В приложениях встречается также уравнение

$$
\ddot{q}-\mu^{2} q=\epsilon f(q, \dot{q}), \quad \mu^{2}>0,
$$

которое получается из (2.1) заменой $\omega=i \mu$. Такое уравнение можно назвать уравнением скатывания. Данный вид имеют, например, уравнение Хиггса [5] и уравнение Фридмана [9], которое описывает динамику тахионного поля в космологии.

По аналогии с методом разложения Боголюбова-Крылова, применявшимся для уравнения колебаний (2.1), будем искать общее решение уравнения скатывания (2.2) в виде разложения

$$
q=a \operatorname{sh} \psi+\epsilon u_{1}(a, \psi)+\epsilon^{2} u_{2}(a, \psi)+\cdots,
$$

где величины $a, \psi$ как функции времени определяются дифференциальными уравнениями

$$
\begin{aligned}
& \dot{a}=\epsilon A(a, \epsilon)=\epsilon A_{1}(a)+\epsilon^{2} A_{2}(a)+\cdots, \\
& \dot{\psi}=\mu+\epsilon B(a, \epsilon)=\mu+\epsilon B_{1}(a)+\epsilon^{2} B_{2}(a)+\cdots .
\end{aligned}
$$

Для построения решения рассмотрим функцию вида $q(t)=\mathfrak{A} \operatorname{sh}(\mu t \mathfrak{m})+\cdots$ и определим параметры $\mathfrak{A}, \mathfrak{m}$ из условия сохранения функциональной формы решения после подстановки в уравнение (1.4). В правой части (1.4) имеем

$$
-\epsilon(\mathfrak{A} \operatorname{sh}(\mu t \mathfrak{m})+\cdots)^{3}=-\epsilon\left(\frac{1}{4} \mathfrak{A}^{3} \operatorname{sh}(3 \mu t \mathfrak{m})+3 \operatorname{sh}(\mu t \mathfrak{m})+\cdots\right) .
$$

В левой части (1.4) имеем

$$
\left(\partial^{2}-\mu^{2}\right)(\mathfrak{A} \operatorname{sh}(\mu t \mathfrak{m})+\cdots)=\mathfrak{A} \mu\left(\mathfrak{m}^{2}-1\right) \operatorname{sh}(\mu t \mathfrak{m})+\cdots .
$$

Таким образом, чтобы получить равенство, необходимо выполнение тождества

$$
\mathfrak{A} \mu^{2}\left(1-\mathfrak{m}^{2}\right)=-\frac{3}{4} \mathfrak{A}^{3} \epsilon,
$$


откуда получаем $\mathfrak{m}^{2}=1+3 \mathfrak{A}^{2} \epsilon / 4 \mu^{2}$. Рассматривая невязку в правой и левой частях (1.4), получаем, что следующее приближение следует взять в виде

$$
q(t)=\mathfrak{A} \operatorname{sh}\left(\mu t\left(1+\frac{3}{8} \frac{\mathfrak{A}^{2} \epsilon}{\mu^{2}}\right)\right)+\mathfrak{A}_{1} \operatorname{sh}(3 \mu t)+\cdots,
$$

и с учетом (2.3) мы получаем

$$
\mathfrak{A}_{1}=-\frac{1}{32} \frac{\mathfrak{A}^{3} \epsilon^{2}}{\mu^{4}} .
$$

Приближение (2.4), (2.5) получено в работе [8].

Описанную процедуру можно продолжить, и оказывается, что для уравнения (1.4) она приводит к представлению решения уравнения с начальным условием $\dot{q}(0)=0$ в виде бесконечной суммы

$$
q(t)=\sum_{n=0}^{\infty} \mathfrak{s}_{n}(t)
$$

где члены $\mathfrak{s}_{n}$, отвечающие модам $n=0,1,2, \ldots$, имеют вид

$$
\begin{aligned}
\mathfrak{s}_{0}(t) & =\mathfrak{A} \operatorname{sh}(\mu t \mathfrak{m}), \\
\mathfrak{s}_{n}(t) & =\mathfrak{A} \mathfrak{a}_{n}(\lambda) \operatorname{sh}((2 n+1) \mu t \mathfrak{m}), \quad n=1,2, \ldots,
\end{aligned}
$$

a $\mathfrak{m}=\mathfrak{m}(\lambda)$ и $\mathfrak{a}_{n}(\lambda)$ являются рядами по безразмерному параметру $\lambda=\mathfrak{A}^{2} \epsilon / \mu^{2}$ :

$$
\begin{aligned}
\mathfrak{m}(\lambda) & =1+\mathfrak{m}_{1} \lambda+\cdots+\mathfrak{m}_{n} \lambda^{n}+\cdots \\
\mathfrak{a}_{n}(\lambda) & =\mathfrak{a}_{n 0} \lambda^{n}+\mathfrak{a}_{n 1} \lambda^{n+1}+\cdots+\mathfrak{a}_{n n} \lambda^{2 n}+\cdots
\end{aligned}
$$

Решение зависит от параметров задачи $\epsilon, \mu$ и характеризуется единственным параметром $\mathfrak{A}$. В этих обозначениях первые члены ряда (2.6) записываются в виде

$$
\begin{aligned}
q(t)= & \mathfrak{A} \operatorname{sh}\left(\mu t\left(1+\frac{3 \mathfrak{A}^{2} \epsilon}{8 \mu^{2}}-\frac{15 \mathfrak{A}^{4} \epsilon^{2}}{256 \mu^{4}}\right)\right)- \\
& -\mathfrak{A}\left(\frac{\mathfrak{A}^{2} \epsilon}{32 \mu^{2}}-\frac{21 \mathfrak{A}^{4} \epsilon^{2}}{1024 \mu^{4}}\right) \operatorname{sh}\left(3 \mu t\left(1+\frac{3 \mathfrak{A}^{2} \epsilon}{8 \mu^{2}}\right)\right)+\frac{21 \mathfrak{A}^{5} \epsilon^{2}}{1024 \mu^{4}} \operatorname{sh}(5 \mu t)+\cdots
\end{aligned}
$$

Этот ряд совпадает с рядом, который получается распространением формул Боголюбова-Крылова для ангармонического осциллятора на случай уравнения Хиггса, и формально может быть получен из разложения метода усреднения (см. ниже формулу (3.1)) заменами $\omega \rightarrow i \mu, A \rightarrow-i \mathfrak{A}$.

Для уравнения Хиггса с трением

$$
\left(\partial^{2}+2 \epsilon h \partial-\omega^{2}\right) q+\epsilon q^{3}=0, \quad q(0)=0,
$$

приближенное решение имеет вид

$$
\begin{aligned}
q(t)= & \mathfrak{A} e^{-\epsilon h t} \operatorname{sh}\left(\mu\left(t-\frac{3 \mathfrak{A}^{2}\left(1-e^{-2 \epsilon h t}\right)}{16 \mu^{2} h}\right)\right)- \\
& -\frac{\mathfrak{A}^{3} \epsilon}{32 \mu^{2}} e^{-3 \epsilon h t} \operatorname{sh}\left(3 \mu\left(t-\frac{3 \mathfrak{A}^{2}\left(1-e^{-2 \epsilon h t}\right)}{16 \mu^{2} h}\right)\right) .
\end{aligned}
$$


Метод, предлагающийся в настоящей статье, можно использовать также при исследовании режима скатывания (не обязательно медленного) для уравнения Фридмана как в локальной, так и нелокальной космологи [7], [8]:

$$
\ddot{q}+\epsilon_{1} \sqrt{\frac{\dot{q}^{2}}{2} \pm \omega^{2} \frac{q^{2}}{2}+\epsilon_{2} V(q)} \pm \omega^{2} q+\epsilon_{2} V^{\prime}(q)=0 .
$$

\section{3. УРАВНЕНИЯ ДЮФФИНГА И ХИГГСА И ЭЛЛИПТИЧЕСКИЕ ФУНКЦИИ}

В этом разделе выписаны точные и приближенные решения простейших нелинейных уравнений теории колебаний и теории скатывания.

3.1. Решения уравнения Дюффинга. Уравнение вида $\ddot{q}+V^{\prime}(q)=0$ имеет интеграл энергии $E=\dot{q}^{2} / 2+V(q)$, и его решение записывается в виде

$$
t=\int \frac{d q}{\sqrt{2(E-V(q))}} .
$$

Если $V(q)$ - многочлен четвертой степени, то последний интеграл выражается через эллиптические функции [12]-[14]. Этот факт приводит к тому, что решение уравнения ангармонического осциллятора (1.3) (уравнения Дюффинга) можно записать как $q(t)=a \operatorname{cn}(\Omega t+b, k)$, где $\operatorname{cn}(u, k)=\mathrm{cn} u$ - эллиптический косинус с модулем $k$. Здесь $a, b, \Omega$ - постоянные, связанные с параметрами уравнения $\omega, \epsilon$ :

$$
\Omega=\sqrt{\omega^{2}+\epsilon a^{2}}, \quad k=\sqrt{\frac{\epsilon a^{2}}{2\left(\omega^{2}+\epsilon^{2}\right)}} .
$$

Функция $\mathrm{cn}(\Omega t+b, k)$ допускает разложение по тригонометрическим функциям:

$$
\operatorname{cn}(\Omega t+b, k)=\frac{2 \pi}{k \mathbf{K}} \sum_{n=1}^{\infty} \frac{\mathfrak{q}^{n-1 / 2}}{1+\mathfrak{q}^{2 n-1}} \cos \left((2 n-1) \frac{\pi(\Omega t+b)}{2 \mathbf{K}}\right),
$$

где $\mathfrak{q}=e^{-\pi \mathbf{K}^{\prime} / \mathbf{K}}$ и $\mathfrak{q}^{\prime}=e^{-\pi \mathbf{K} / \mathbf{K}^{\prime}}$, a $\mathbf{K}=\mathbf{K}(k)$ и $\mathbf{K}^{\prime}=\mathbf{K}^{\prime}(k)$ - полные эллиптические интегралы первого рода. Из этого представления можно получить асимптотическое разложение при малых $\epsilon$, которое получается с помощью метода усреднения Крылова-Боголюбова [2]:

$$
q(t)=A \sin \left(\omega t\left(1+\frac{3 A^{2} \epsilon}{8 \omega^{2}}\right)\right)-\frac{A^{3} \epsilon}{32 \omega^{2}} \sin \left(3 \omega t\left(1+\frac{3 A^{2} \epsilon}{8 \omega^{2}}\right)\right)+\cdots,
$$

где $A$ - произвольный вещественный параметр и взято начальное условие $q(0)=0$.

3.2. Решение уравнения Хиггса. Уравнение (1.4) в зависимости от начальных данных $q(0)=q_{0}$ и $\dot{q}(0)=v_{0}$ имеет три типа решений, которые определяются знаком энергии

$$
E=\frac{1}{2} v_{0}^{2}-\frac{1}{2} \mu^{2} q_{0}^{2}+\frac{1}{4} \epsilon q_{0}^{4} .
$$

1. Если энергия $E>0$, то мы имеем решение "в 2-х впадинах" потенциальной энергии:

$$
q(t)=a \operatorname{cn}(\Omega t+b, k),
$$


где

$$
a^{2}=\frac{\mu^{2}}{\epsilon}\left(1+\sqrt{1+\frac{4 \epsilon E}{\mu^{4}}}\right), \quad \Omega^{2}=\mu^{2} \sqrt{1+\frac{4 \epsilon E}{\mu^{4}}}, \quad k^{2}=\frac{1}{2}+\frac{1}{2} \frac{1}{\sqrt{1+4 \epsilon E / \mu^{4}}} .
$$

Отметим также, что

$$
\left(k^{\prime}\right)^{2}=\frac{1}{2}-\frac{1}{2} \frac{1}{\sqrt{1+4 \epsilon E / \mu^{4}}} .
$$

Параметр $b$ определяется из условия $q(0)=a \mathrm{cn}(b, k)$. Решение параметризовано величинами $q_{0}, v_{0}$ или $E, b$. Мы рассмотрим случай специальных начальных данных: $q(0)=0$. При этом $b=-\mathbf{K}$ и

$$
q(t)=a \operatorname{cn}(\Omega t-\mathbf{K}, k) .
$$

Для этого решения

$$
v \equiv \dot{q}(0)=\frac{a \mu k^{\prime}}{\sqrt{1-2\left(k^{\prime}\right)^{2}}}
$$

а энергия $E$ связана с параметром $k^{\prime}$ :

$$
\frac{E \epsilon}{\mu^{4}}=\frac{\mu^{4}\left(k^{\prime}\right)^{2}\left(1-\left(k^{\prime}\right)^{2}\right)}{\left(2\left(k^{\prime}\right)^{2}-1\right)^{2}} .
$$

Отметим также связь параметра $а$ с величинами $\epsilon$ и $k^{\prime}$,

$$
a^{2}=\frac{\mu^{2}}{\epsilon}\left(1+\frac{1}{1-2\left(k^{\prime}\right)^{2}}\right),
$$

а также связь $\Omega$ и параметров $\mu, k^{\prime}$ :

$$
\Omega^{2}=\frac{\mu^{2}}{1-2\left(k^{\prime}\right)^{2}} .
$$

2. Если энергия $E=0$, то мы имеем решение "в 2-х впадинах" с периодом $T=\infty$ :

$$
q(t)=\mu \sqrt{\frac{2}{\epsilon}} \frac{1}{\operatorname{ch}(\mu t+b)},
$$

где параметр $b$ находится из начального условия

$$
q(0)=\mu \sqrt{\frac{2}{\epsilon}} \frac{1}{\operatorname{ch} b} .
$$

3. Если энергия $E<0$, то мы имеем решение "в одной впадине", например правой:

$$
q(t)=a \operatorname{dn}(\Omega t+b, k)
$$

где

$$
\begin{gathered}
a^{2}=\frac{\mu^{2}}{\epsilon}\left(1+\sqrt{1+\frac{4 \epsilon E}{\mu^{4}}}\right), \quad \Omega^{2}=a^{2} \frac{\epsilon}{2}=\frac{\mu^{2}}{2}\left(1+\sqrt{1+\frac{4 \epsilon E}{\mu^{4}}}\right), \\
k^{2}=2-\frac{2 \mu^{2}}{\epsilon a^{2}}=2-\frac{2}{1+\sqrt{1+4 \epsilon E / \mu^{4}}} .
\end{gathered}
$$


Параметр $b$ определяется соотношением $q(0)=a \operatorname{dn}(b, k)$. Энергия в этом случае может быть записана через параметр $k^{\prime}$ :

$$
E=-\frac{\mu^{4}\left(k^{\prime}\right)^{2}}{\epsilon\left(\left(k^{\prime}\right)^{2}+1\right)^{2}} .
$$

В случае специального начального условия $v_{0}=0$ имеем $q(t)=a \operatorname{dn}(\Omega t-\mathbf{K}, k)$. Режим скатывания соответствует движению около "точки поворота", ближайшей к вершине.

\section{4. РАЗЛОЖЕНИЕ ЭЛЛИПТИЧЕСКИХ ФУНКЦИЙ}

Рассмотрим решение (3.4). Оно является периодическим с периодом $T=4 \mathbf{K} / \Omega$, где $\mathbf{K}=\mathbf{K}(k)$. Покажем, что при $|t|<T / 2$ решение допускает разложение в ряд (2.9) по гиперболическим функциям и первые члены разложения (2.6) действительно являются достаточно точным приближением к решению (3.2) при $b=-\mathbf{K}$. Важность этого замечания связана с тем, что для более сложных потенциалов или в задаче с трением мы можем непосредственно применять гиперболический аналог метода Крылова-Боголюбова, в то время как точные решения в этих случаях неизвестны (см., например, задачу с трением (2.10)).

Нам понадобится разложение эллиптической функции в окрестности точки, координата которой равна четверти полупериода, взятой со знаком минус, т. е. функции $\operatorname{cn}(u-\mathbf{K}, k)$, при малых значениях $k^{\prime}$. При $k^{\prime} \rightarrow 0$ полный эллиптический интеграл $\mathbf{K}=\mathbf{K}\left(\sqrt{1-\left(k^{\prime}\right)^{2}}\right)$ имеет следующую асимптотику:

$$
\mathbf{K} \approx \ln \frac{4}{k^{\prime}}+\frac{\left(k^{\prime}\right)^{2}}{4} \ln \frac{4}{\left(k^{\prime}\right)^{2}}-\frac{\left(k^{\prime}\right)^{2}}{4}+\cdots .
$$

Нас интересует предел $k^{\prime} \rightarrow 0$, поскольку именно в этом пределе согласно (3.2) параметр $\epsilon \rightarrow 0$ :

$$
k^{\prime}=\frac{\sqrt{E \epsilon}}{\mu^{2}}\left(1-\frac{3}{2} \frac{E \epsilon}{\mu^{4}}+O\left(\epsilon^{2}\right)\right) .
$$

В силу элементарного неравенства

$$
\frac{1}{2 y}\left(1-\frac{1}{\sqrt{1+y}}\right)<\frac{1}{2} \quad \text { при } \quad 0 \leqslant y<\infty
$$

имеет место оценка

$$
\left(k^{\prime}\right)^{2} \leqslant \frac{2 \epsilon E}{\mu^{4}}
$$

и, следовательно, разложение по $k^{\prime}$ соответствует разложению по $\sqrt{\epsilon E}$.

По формуле сложения для элиптических функций с учетом равенств sn $\mathbf{K}=1$, cn $\mathbf{K}=0, \operatorname{dn} \mathbf{K}=k^{\prime}$ мы имеем

$$
\begin{aligned}
\operatorname{cn}(u-\mathbf{K}) & =\frac{\operatorname{cn} u \operatorname{cn} \mathbf{K}+\operatorname{sn} u \operatorname{sn} \mathbf{K} \operatorname{dn} u \operatorname{dn} \mathbf{K}}{1-k^{2} \operatorname{sn}^{2} u \operatorname{sn}^{2}(\mathbf{K})}= \\
& =k^{\prime} \frac{\operatorname{sn} u \operatorname{dn} u}{1-k^{2} \operatorname{sn}^{2} u}=k^{\prime} \frac{\operatorname{sn} u \operatorname{dn} u}{\operatorname{dn}^{2} u}=k^{\prime} \frac{\operatorname{sn} u}{\operatorname{dn} u} .
\end{aligned}
$$


4.1. Теория возмущений. Рассмотрим разложение эллиптической функции (4.2), представляющей решение, в ряд по малому параметру $k^{\prime}$ (т. е. по параметру $\epsilon$ ) и сравним первые члены ряда теории возмущений с точным решением. При малых $k^{\prime}$ имеют место следующие разложения [14]:

$$
\begin{aligned}
& \operatorname{sn}(u, k)=\operatorname{th} u+\frac{1}{4}\left(k^{\prime}\right)^{2}(\operatorname{sh} u \operatorname{ch} u-u) \frac{1}{\operatorname{ch}^{2} u}+\cdots, \\
& \operatorname{dn}(u, k)=\frac{1}{\operatorname{ch} u}+\frac{1}{4}\left(k^{\prime}\right)^{2}(\operatorname{sh} u \operatorname{ch} u+u) \frac{\operatorname{sh} u}{\operatorname{ch}^{2} u}+\cdots .
\end{aligned}
$$

Следовательно, при малых $k^{\prime}$

$$
\frac{\operatorname{sn}(u, k)}{\operatorname{dn}(u, k)}=\operatorname{sh} u+\frac{1}{4}\left(k^{\prime}\right)^{2}\left(\operatorname{sh} u-\operatorname{sh}^{2} u\right)-\frac{1}{4}\left(k^{\prime}\right)^{2} u\left(\frac{1}{\operatorname{ch} u}-\operatorname{th} u\right)+\cdots,
$$

и мы получаем следующее разложение:

$$
\operatorname{cn}(u-\mathbf{K}, k)=k^{\prime} \mathfrak{c}_{0}(u)+\left(k^{\prime}\right)^{3} \mathfrak{c}_{1}(u)+\cdots,
$$

где

$$
\begin{aligned}
& \mathfrak{c}_{0}(u)=\operatorname{sh} u \\
& \mathfrak{c}_{1}(u)=\frac{1}{4}\left(\operatorname{sh} u-\operatorname{sh}^{2} u\right)-\frac{1}{4} u\left(\frac{1}{\operatorname{ch} u}-\operatorname{th} u\right) .
\end{aligned}
$$

В этом разложении имеется секулярный член $\left(k^{\prime}\right)^{3}$, однако, в отличие от тригонометрического случая, здесь при достаточно больших значениях переменной $u$ доминирует член $\left(k^{\prime}\right)^{3} \mathrm{sh}^{2} u / 4$. В следующем пункте построена модифицированная теория возмущений с использованием разложения по гиперболическим функциям, которая более точно описывает решение при достаточно больших значениях $u$. Интересно отметить, что, как будет показано ниже, в нашей модифицированной теории возмущений важный вклад дает член $-\left(k^{\prime}\right)^{3} \operatorname{sh}(3 u) / 16$, который с ростом $u$ растет быстрее, чем член $\left(k^{\prime}\right)^{3} \operatorname{sh}^{2} u / 4$ из теории возмущений.

4.2. Модифицированная теория возмущений. Покажем, что

$$
\operatorname{cn}(u-\mathbf{K}, k)=\sum_{n=0}^{\infty}(-1)^{n} \mathcal{A}_{n} \operatorname{sh}\left((2 n+1) u^{\prime}\right)
$$

где

$$
\mathcal{A}_{n}=\frac{\pi}{k \mathbf{K}^{\prime}} \frac{1}{\operatorname{ch}\left((2 n+1) \rho^{\prime}\right)}, \quad u^{\prime}=\frac{\pi u}{2 \mathbf{K}^{\prime}}, \quad \rho^{\prime}=\frac{\pi \mathbf{K}}{2 \mathbf{K}^{\prime}},
$$

и ряд в правой части (4.6) сходится при $|\operatorname{Re} u|<\mathbf{K}$ и $-1<k<1$. Для доказательства формулы (4.6) используем соотношение (4.2) и заметим, что имеет место представление [12]

$$
\frac{\operatorname{sn} u}{\operatorname{dn} u}=\frac{\pi}{k k^{\prime} \mathbf{K}^{\prime}}\left\{\frac{\operatorname{sh} u^{\prime}}{\operatorname{ch} \rho^{\prime}}-\frac{\operatorname{sh}\left(3 u^{\prime}\right)}{\operatorname{ch}\left(3 \rho^{\prime}\right)}+\frac{\operatorname{sh}\left(5 \rho^{\prime}\right)}{\operatorname{ch}\left(5 u^{\prime}\right)}+\cdots\right\} .
$$

Умножение последнего равенства на $k^{\prime}$ и дает формулу (4.6).

6 Теоретическая и математическая физика, т. 172, № 1, 2012 г. 

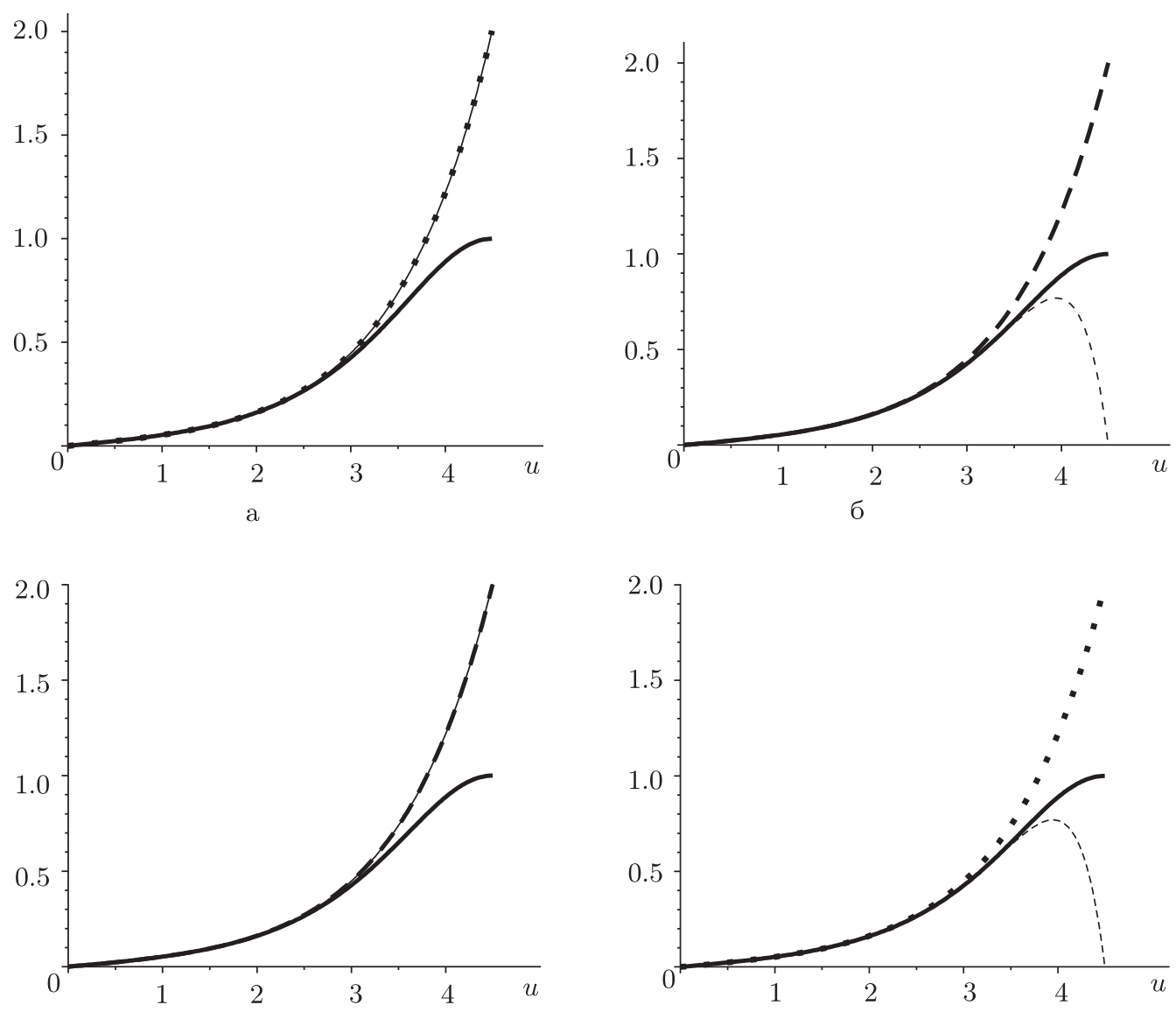

B

$\Gamma$

Рис. 1. Сравнение первых двух порядков обычной (см. формулы (4.4), (4.5)) и модифицированной (см. формулы $(4.7),(4.8))$ теории возмущений с точным решением $\operatorname{cn}(u,-\mathbf{K}), k=0.999$, и между собой. Представлены первое (тонкая сплошная линия) и второе (пунктир) приближения в рамках обычной теории возмущений (а); первое (жирная штриховая линия) и второе (тонкая штриховая линия) приближения в рамках модифицированной теории возмущений (б); первые приближения в рамках обычной (тонкая сплошная линия) и модифицированной (жирная штриховая линия) теории возмущений (в); вторые приближения в рамках обычной (пунктир) и модифицированной (тонкая штриховая линия) теории возмущений (г). На всех рисунках жирная сплошная линия отвечает точному решению.

Мы будем рассматривать приближения

$$
\begin{aligned}
\mathfrak{C}^{(0)}(u) & =\mathcal{A} \operatorname{sh} u^{\prime}, \quad A=\mathcal{A}_{0}, \\
\mathfrak{C}^{(1)}(u) & =\mathcal{A} \operatorname{sh} u^{\prime}-\mathcal{A}_{1} \operatorname{sh}\left(3 u^{\prime}\right), \\
\ldots \ldots \ldots \ldots \ldots \ldots \ldots \ldots \ldots & \ldots \ldots \ldots \\
\mathfrak{C}^{(n)}(u) & =\mathcal{A} \operatorname{sh} u^{\prime}-\mathcal{A}_{1} \operatorname{sh}\left(3 u^{\prime}\right)+\cdots+(-1)^{n} \mathcal{A}_{n} \operatorname{sh}\left((2 n+1) u^{\prime}\right) .
\end{aligned}
$$


Отметим, что $u^{\prime}$ и $\mathcal{A}_{2 n+1}, n=0,1,2$, имеют следующие разложения в ряды по $\left(k^{\prime}\right)^{2}$ :

$$
u^{\prime}=\frac{\pi}{2 \mathbf{K}^{\prime}} u \approx\left(1-\frac{1}{4}\left(k^{\prime}\right)^{2}+\cdots\right) u
$$

и

$$
\begin{gathered}
\mathcal{A}=k^{\prime}+\frac{7}{16}\left(k^{\prime}\right)^{3}+\frac{79}{256}\left(k^{\prime}\right)^{5}+O\left(k^{\prime}\right)^{7}, \\
\mathcal{A}_{1}=-\left(\frac{1}{16}\left(k^{\prime}\right)^{3}+\frac{1}{16}\left(k^{\prime}\right)^{5}+O\left(k^{\prime}\right)^{7}\right), \quad \mathcal{A}_{3}=\frac{1}{4096}\left(k^{\prime}\right)^{7}+O\left(k^{\prime}\right)^{9},
\end{gathered}
$$

однако в рамках принятых обозначений мы не используем эти разложения.

На рис. 1 приведены графики первых двух слагаемых разложений по обычной и модифицированной теории возмущений в сравнении с точным решением $\mathrm{cn}(u,-\mathbf{K})$ при $k=0.999$, которое на всех рисунках изображено жирной сплошной линией. На рис. 1а первое приближение (4.4) по обычной теории возмущения изображено тонкой сплошной линией, второе приближение (4.5) - пунктиром. Видно, что первое и второе приближения обычной теории возмущений почти совпадают друг с другом, и второе приближение практически не является улучшением первого приближения. На рис. $1 б$ изображены два первых порядка приближения по модифицированной теории возмущений: первый порядок (4.7) - жирная штриховая линия и второй порядок (4.8) - тонкая штриховая линия. Рис. 1в приведен для сравнения приближений первого порядка в обычной (тонкая сплошная линия) и модифицированной (жирная штриховая линия) теории возмущений. Аналогичное сравнение для второго порядка представлено на рис. 1г: обычная теория возмущений - пунктирная линия, модифицированная - тонкая штриховая линия. Видно, что первые приближения, вычисленные в рамках обычной и модифицированной теории возмущений, практически совпадают друг с другом, однако вторые приближения существенно различаются. Второе приближение, вычисленное по модифицированной теории возмущений, ближе к точному ответу по сравнению со вторым приближением обычной теории возмущений.

Следующие члены разложения представлены на рис. 2.

\section{3. Оценка модифицированного ряда для эллиптического косинуса.} Можно доказать следующую лемму, дающую оценку сходимости ряда (4.6).

Лемма 1. Пусть $\mu>0,0<c<1 / 2$ и $\Omega$ задано формулой (3.5). Тогда при всех

$$
0 \leqslant t \leqslant \frac{c}{\mu \sqrt{2}} \ln \frac{1}{k^{\prime}}, \quad 0 \leqslant k^{\prime} \leqslant 1 / 2
$$

выполняются неравенства

$$
\begin{aligned}
& \left|\operatorname{cn}(\Omega t-\mathbf{K}, k)-\mathfrak{C}^{(0)}(\Omega t)\right| \leqslant \frac{4}{k}\left(k^{\prime}\right)^{3(1-c)}, \\
& \left|\operatorname{cn}(\Omega t-\mathbf{K}, k)-\mathfrak{C}^{(1)}(\Omega t)\right| \leqslant \frac{4}{k}\left(k^{\prime}\right)^{5(1-c)}
\end{aligned}
$$

здесъ $\mathfrak{C}^{(0)}(u)$ и $\mathfrak{C}^{(1)}(u)$ означают приближения, введенные формулами (4.7) и (4.8). 


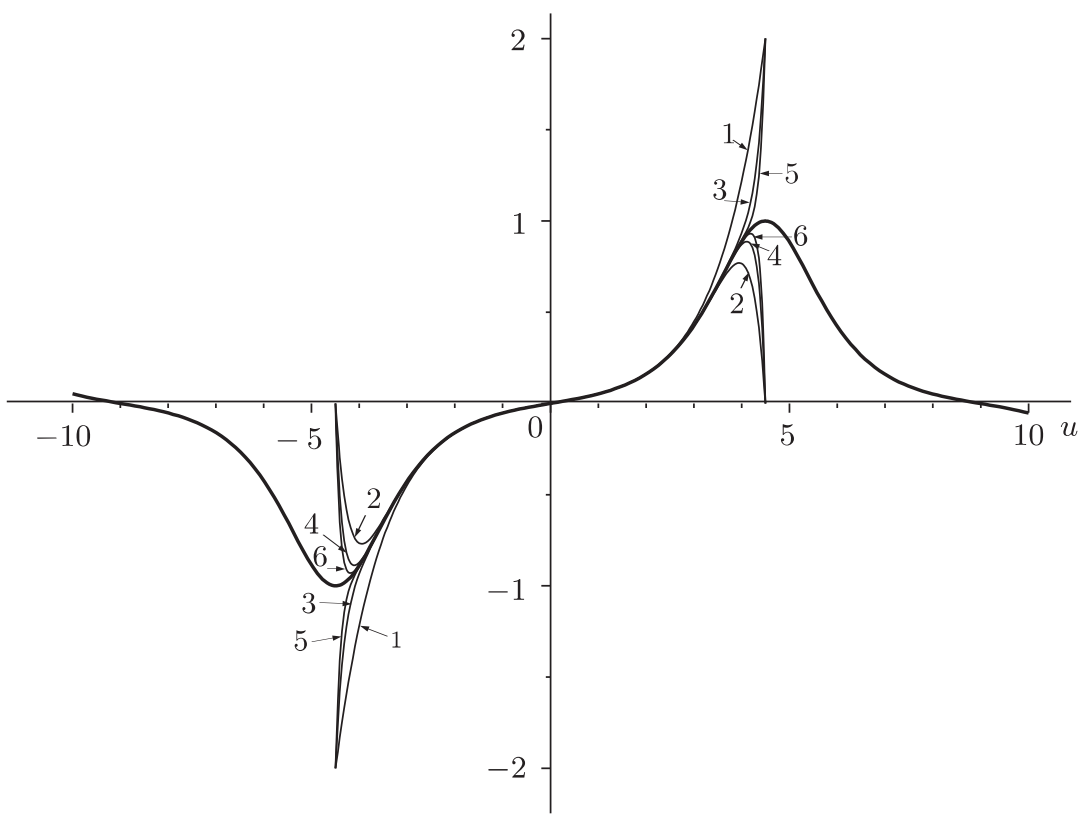

a

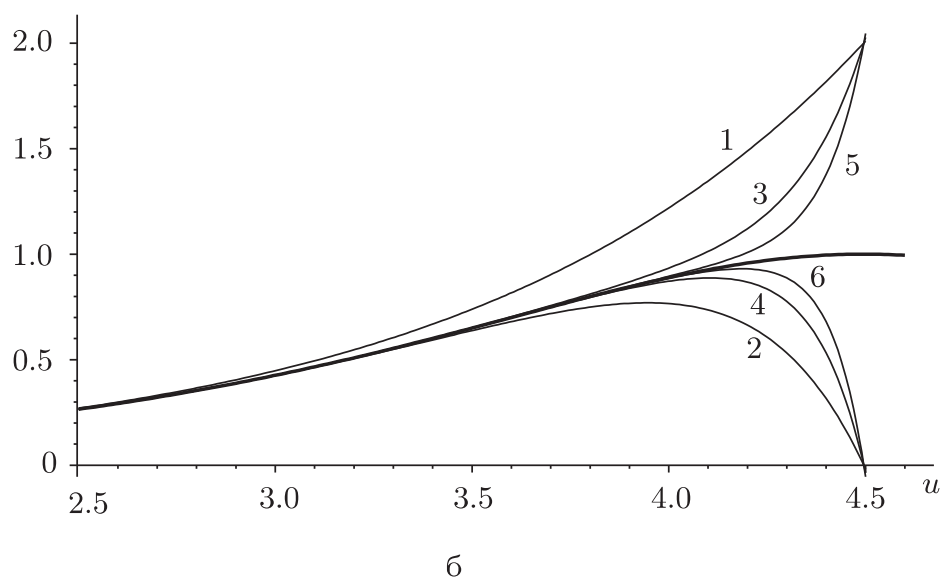

Рис. 2. Приближения более высоких порядков, вычисленные по формуле (4.6): общий вид (а) и поведение решений в окрестности максимума (б); номер кривой соответствует порядку приближения. Жирная сплошная линия - функция $\operatorname{cn}(u-\mathbf{K}, k)$ при $k=0.999$.

ЗАмечаниЕ 1. Нетривиальность оценок (4.11) по сравнению с утверждением о сходимости ряда (4.6) при $|\operatorname{Re} u|<\mathbf{K}$ и $-1<k<1$ связана с тем, что эти оценки в силу (4.10) позволяют рассматривать предел $t \rightarrow \infty$ при $k^{\prime} \rightarrow 0$. 


\section{5. ПРИБЛИЖЕНИЯ ТОЧНОГО РЕШЕНИЯ}

В этом разделе приведены приближения точного решения, в которых используются разложения (4.7)-(4.9).

5.1. Оценка точности приближения, содержащего одну моду. Рассмотрим приближение точного решения (3.4), используя его представление в виде ряда (4.6). Имеем

$$
q(t)=a \operatorname{cn}(\Omega t-\mathbf{K}, k)=\sum_{n=0}^{\infty} s_{n}(t),
$$

где введены обозначения $(n=1,2, \ldots)$

$$
\begin{aligned}
& s_{0}(t)=A \operatorname{sh} \frac{\pi \Omega t}{2 \mathbf{K}^{\prime}}, s_{n}(t)=(-1)^{n} A_{n} \operatorname{sh} \frac{(2 n+1) \pi \Omega t}{2 \mathbf{K}^{\prime}}, \\
& A=a \frac{\pi}{k \mathbf{K}^{\prime}} \frac{1}{\operatorname{ch}\left(\pi \mathbf{K} / 2 \mathbf{K}^{\prime}\right)}, \quad A_{n}=a \frac{\pi}{k \mathbf{K}^{\prime}} \frac{1}{\operatorname{ch}\left((2 n+1) \pi \mathbf{K} / 2 \mathbf{K}^{\prime}\right)} .
\end{aligned}
$$

Определим приближения, содержащие $n=0,1,2, \ldots$ мод:

$$
q_{0}(t)=s_{0}(t), \quad q_{n}(t)=s_{0}(t)+s_{1}(t)+\cdots+s_{n}(t)
$$

(заметим, что мы не указываем явно зависимость функций от $\left.\left(k^{\prime}\right)^{2}\right)$.

Из леммы 1 и оценки (4.1) вытекает следующая

TeOpema 1. Пусть $0<c<1 / 2$. Тогда при всех

$$
0 \leqslant t \leqslant \frac{c}{\mu 2 \sqrt{2}} \ln \frac{\mu^{4}}{2 \epsilon E}
$$

выполнена оценка

$$
\left|q(t)-q_{1}(t)\right| \leqslant \frac{8 \sqrt{2 E}}{k \mu}\left(\frac{2 \epsilon E}{\mu^{4}}\right)^{2-5 c / 2} .
$$

\section{2. Гиперболический аналог модифицированной теории возмущений.} Цель последующих рассуждений - дать оценку приближения, получающегося из представления (2.9). Для этого заметим, что в приближении, заданном формулами (5.2)-(5.4), как постоянные $A_{i}$, так и аргументы гиперболических синусов являются рядами по $\left(k^{\prime}\right)^{2}$ и, следовательно, по $\epsilon$.

5.2.1. Переразложение ряда для эллиптического косинуса. Имеет место следующее представление, позволяющее от ряда (5.1) перейти к ряду (2.6): решение (5.1) может быть представлено в виде

$$
q(t)=a \operatorname{cn}(\Omega t-\mathbf{K}, k)=\sum_{n=0}^{\infty}(-1)^{n} A \bar{a}_{n}(\lambda) \operatorname{sh}((2 n+1) \mu t \overline{\mathbf{\Omega}}(\lambda)) .
$$

Здесь $\lambda=A^{2} \epsilon / \mu^{2}$, а $\bar{a}_{n}(\lambda)$ и $\overline{\boldsymbol{\Omega}}(\lambda)$ выражаются через функции

$$
\begin{gathered}
a_{n}\left(\left(k^{\prime}\right)^{2}\right)=\frac{\operatorname{ch}\left(\pi \mathbf{K} / 2 \mathbf{K}^{\prime}\right)}{\operatorname{ch}\left((2 n+1) \pi \mathbf{K} / 2 \mathbf{K}^{\prime}\right)}, \quad n=1,2, \ldots, \quad a_{0}\left(\left(k^{\prime}\right)^{2}\right)=1, \\
\boldsymbol{\Omega}\left(\left(k^{\prime}\right)^{2}\right)=\frac{1}{\mu} \frac{\pi \Omega}{2 \mathbf{K}^{\prime}}=\frac{1}{\sqrt{1-2\left(k^{\prime}\right)^{2}}} \frac{\pi}{2 \mathbf{K}^{\prime}}
\end{gathered}
$$


при помощи замены переменных

$$
\begin{gathered}
\bar{a}_{n}(\lambda)=a_{n}\left(F^{-1}(\lambda)\right), \quad n=1,2, \ldots, \quad a_{0}(\lambda)=1, \\
\bar{\Omega}(\lambda)=\boldsymbol{\Omega}\left(F^{-1}(\lambda)\right),
\end{gathered}
$$

где функция $F^{-1}(\lambda)$ определяется из соотношения $\lambda=F\left(\left(k^{\prime}\right)^{2}\right)$,

$$
F\left(\left(k^{\prime}\right)^{2}\right)=\left(1+\frac{1}{1-2\left(k^{\prime}\right)^{2}}\right)\left(\frac{\pi / k \mathbf{K}^{\prime}}{\operatorname{ch}\left(\pi \mathbf{K} / 2 \mathbf{K}^{\prime}\right)}\right)^{2}
$$

В низшем порядке

$$
\lambda=2\left(k^{\prime}\right)^{2}+\frac{15}{4}\left(k^{\prime}\right)^{4}+O\left(k^{\prime}\right)^{6} .
$$

Доказательство представления очевидно. Неочевидным фактом является совпадение таким образом построенного ряда с рядом (2.6) (см. ниже лемму 2).

\section{3. Приближения, содержащие $n$ мод.}

5.3.1. Приближения, содержащие $n$ мод, как ряды по $k^{\prime}$. Рассмотрим следующие ряды по $\left(k^{\prime}\right)^{2}$ :

$$
\begin{gathered}
\boldsymbol{\Omega}=\frac{1}{\sqrt{1-2\left(k^{\prime}\right)^{2}}} \frac{\pi}{2 \mathbf{K}^{\prime}}=1+\left(k^{\prime}\right)^{2} \boldsymbol{\Omega}_{1}+\left(k^{\prime}\right)^{4} \boldsymbol{\Omega}_{2}+\cdots, \\
\frac{\operatorname{ch}\left(\pi \mathbf{K} / 2 \mathbf{K}^{\prime}\right)}{\operatorname{ch}\left((2 i+1) \pi \mathbf{K} / 2 \mathbf{K}^{\prime}\right)}=\left(k^{\prime}\right)^{2 i} l_{i 0}+\left(k^{\prime}\right)^{2(i+1)} l_{i 1}+\cdots .
\end{gathered}
$$

Приведем явный вид младших коэффициентов:

$$
\boldsymbol{\Omega}_{1}=\frac{3}{4}, \quad \boldsymbol{\Omega}_{2}=\frac{75}{64}, \quad l_{10}=\frac{1}{16}, \quad l_{11}=\frac{9}{256} .
$$

Используя приближения

$$
\begin{aligned}
& \boldsymbol{\Omega}^{(n)}=1+\left(k^{\prime}\right)^{2} \boldsymbol{\Omega}_{1}+\cdots+\left(k^{\prime}\right)^{2 n} \boldsymbol{\Omega}_{n}, \\
& A_{i}^{(n)}=A\left(\left(k^{\prime}\right)^{2 i} l_{i 0}+\left(k^{\prime}\right)^{2(i+1)} l_{i 1}+\cdots+\left(k^{\prime}\right)^{2(i+n)} l_{i n}\right),
\end{aligned}
$$

мы получим следующие приближения для отдельных слагаемых в ряду (5.1):

$$
\begin{aligned}
& s_{0}^{(0)}(t)=A \operatorname{sh}(\mu t), \\
& s_{0}^{(1)}(t)=A \operatorname{sh}\left(\mu t\left(1+\left(k^{\prime}\right)^{2} \boldsymbol{\Omega}_{1}\right)\right), \ldots, \\
& s_{0}^{(n)}(t)=A \operatorname{sh}\left(\mu t\left(1+\left(k^{\prime}\right)^{2} \boldsymbol{\Omega}_{1}+\cdots+\left(k^{\prime}\right)^{2 n} \boldsymbol{\Omega}_{n}\right)\right) .
\end{aligned}
$$

Заметим, что в рамках принятых обозначениях мы не разлагаем $A$ в ряд по $\left(k^{\prime}\right)^{2}$.

Аналогично,

$$
s_{i}^{(n, j)}(t)=(-1)^{i} A_{i}^{(n)} \operatorname{sh}\left((2 i+1) \mu t \Omega^{(j)}\right)
$$

или, в более явном виде,

$$
\begin{aligned}
s_{i}^{(n, j)}(t)= & (-1)^{i} A\left(\left(k^{\prime}\right)^{2 i} l_{i, 0}+\cdots+\left(k^{\prime}\right)^{2(n+i)} l_{i n}\right) \times \\
& \times \operatorname{sh}\left((2 i+1) \mu t\left(1+\left(k^{\prime}\right)^{2} \boldsymbol{\Omega}_{1}+\cdots+\left(k^{\prime}\right)^{2 j} \boldsymbol{\Omega}_{j}\right)\right) .
\end{aligned}
$$


В соответствии с разложениями (5.6) и (5.7) имеем следующие приближения $\mathrm{K} s_{1}(t)$ :

$$
\begin{aligned}
& s_{1}^{(0,0)}(t)=-A\left(k^{\prime}\right)^{2} l_{10} \operatorname{sh}(3 \mu t), \\
& s_{1}^{(0,1)}(t)=-A\left(k^{\prime}\right)^{2} l_{10} \operatorname{sh}\left(3 \mu t\left(1+\left(k^{\prime}\right)^{2} \boldsymbol{\Omega}_{1}\right)\right), \\
& s_{1}^{(1,2)}(t)=-A\left(\left(k^{\prime}\right)^{2} l_{10}\left(k^{\prime}\right)^{4} l_{11}\right) \operatorname{sh}\left(3 \mu t\left(1+\left(k^{\prime}\right)^{2} \boldsymbol{\Omega}_{1}\left(k^{\prime}\right)^{4} \boldsymbol{\Omega}_{2}\right)\right) .
\end{aligned}
$$

Мы также будем использовать обозначения

$$
\begin{aligned}
& s_{i}^{(\text {exact }, j)}(t)=(-1)^{i} A_{i} \operatorname{sh}\left((2 i+1) \mu t\left(1+\left(k^{\prime}\right)^{2} \boldsymbol{\Omega}_{1}+\cdots+\left(k^{\prime}\right)^{2 j} \boldsymbol{\Omega}_{j}\right)\right), \\
& s_{i}^{(n, \text { exact })}(t)=(-1)^{i} A\left(\left(k^{\prime}\right)^{2 i} l_{i 0}+\cdots+\left(k^{\prime}\right)^{2(n+i)} l_{i n}\right) \operatorname{sh}((2 i+1) \mu t \boldsymbol{\Omega}) .
\end{aligned}
$$

5.3.2. Приближения, содержащие $n$ мод, как ряды по $\lambda$. Чтобы получить переразложение ряда (5.1), аналогичное (2.6), нам надо разложить $\Omega$ и $A_{i} / A$ в ряды по $\epsilon$ :

$$
\begin{aligned}
& \boldsymbol{\Omega}=\mu\left[1+\frac{A^{2} \epsilon}{\mu^{2}} \bar{\Omega}_{1}+\left(\frac{A^{2} \epsilon}{\mu^{2}}\right)^{2} \bar{\Omega}_{2}+\cdots\right], \\
& A_{i}=A\left[\left(\frac{A^{2} \epsilon}{\mu^{2}}\right)^{i} \bar{l}_{i 0}+\left(\frac{A^{2} \epsilon}{\mu^{2}}\right)^{i+1} \bar{l}_{i 1}+\cdots\right],
\end{aligned}
$$

где

$$
\bar{\Omega}_{1}=\frac{3}{8}, \quad \bar{l}_{10}=\frac{1}{32} .
$$

Будем также использовать обозначения

$$
\begin{aligned}
& \bar{\Omega}^{(n)}=\mu\left[1+\frac{A^{2} \epsilon}{\mu^{2}} \bar{\Omega}_{1}+\left(\frac{A^{2} \epsilon}{\mu^{2}}\right)^{2} \bar{\Omega}_{2}+\cdots+\left(\frac{A^{2} \epsilon}{\mu^{2}}\right)^{n} \bar{\Omega}_{n}\right] \\
& \bar{A}_{i}^{(n)}=A\left[\left(\frac{A^{2} \epsilon}{\mu^{2}}\right)^{i} \bar{l}_{i 0}+\left(\frac{A^{2} \epsilon}{\mu^{2}}\right)^{i+1} \bar{l}_{i 1}+\cdots+\left(\frac{A^{2} \epsilon}{\mu^{2}}\right)^{i+n} \bar{l}_{i n}\right] .
\end{aligned}
$$

В низших порядках имеем следующие приближения:

$$
\begin{aligned}
\bar{s}_{1}^{(0)}(t) & =A \operatorname{sh}(\mu t), \\
\bar{s}_{0}^{(1)}(t) & =A \operatorname{sh}\left(\mu t\left(1+\frac{A^{2} \epsilon}{\mu^{2}} \bar{\Omega}_{1}\right)\right), \\
\bar{s}_{1}^{(0,1)}(t) & =\frac{A^{3} \epsilon}{\mu^{2}} \bar{l}_{1} \operatorname{sh}\left(3 \mu t\left(1+\frac{A^{2} \epsilon}{\mu^{2}} \bar{\Omega}_{1}\right)\right), \\
\bar{s}_{1}^{(1,2)}(t) & =A\left(\frac{A^{2} \epsilon}{\mu^{2}} \bar{l}_{10}+\left(\frac{A^{2} \epsilon}{\mu^{2}}\right)^{2} \bar{l}_{11}\right) \operatorname{sh}\left(3 \mu t\left(1+\frac{A^{2} \epsilon}{\mu^{2}} \bar{\Omega}_{1}+\left(\frac{A^{2} \epsilon}{\mu^{2}}\right)^{2} \bar{\Omega}_{2}\right)\right),
\end{aligned}
$$

где $\bar{\Omega}_{1}$ и $\bar{l}_{10}$ задаются формулами (5.11). Черта над $s_{i}$ означает, что мы берем разложение по $\epsilon$.

Имеет место

ЛЕмма 2. При отождествлении амплитуд в (5.3) и (2.9), m.е. при $\mathfrak{A}=A$, справедливы равенства $\mathfrak{a}_{n k}=\bar{l}_{n k} u \mathfrak{m}_{i}=\overline{\boldsymbol{\Omega}}_{i}$. 
Данная лемма позволяет утверждать, что при $\mathfrak{A}=A$

$$
\begin{array}{rlrl}
\mathfrak{s}_{0}^{(i)}(t) & =\bar{s}_{0}^{(i)}(t), \quad i & =1,2, \ldots, \\
\mathfrak{s}_{n}^{(i, j)}(t) & =\bar{s}_{n}^{(i, j)}(t), \quad n=1, \ldots, i, \quad j=0,1, \ldots,
\end{array}
$$

если амплитуды в (2.7) и (5.3) одинаковы.

5.3.3. Приближения, содержащие одну моду. Точному выражению $q_{0}(t)$ для приближения, содержащего одну моду (см. формулу (5.4)), сопоставим в соответствии с формулами (5.8) и (5.9) низшие приближения следующего вида:

$$
\bar{s}_{0}^{(0)}(t)=A \operatorname{sh}(\mu t), \quad \bar{s}_{0}^{(1)}(t)=A \operatorname{sh}\left(\mu t\left(1+\frac{3 A^{2}}{8 \mu^{2}} \epsilon\right)\right) .
$$

В данных выражениях по сравнению с (5.2) присутствуют гиперболические синусы с другими аргументами. Разность аргументов аргументов дается выражениями

$$
\begin{aligned}
\Delta_{0} & =\mu t \delta_{0}=\mu t\left(1-\frac{\pi}{2 \mathbf{K}^{\prime}} \frac{1}{\sqrt{1-2\left(k^{\prime}\right)^{2}}}\right) \\
\Delta_{1} & =\mu t \delta_{1}=\mu t\left(\left(1+\frac{3 A^{2}}{8 \mu^{2}} \epsilon\right)-\frac{\pi \Omega}{2 \mu \mathbf{K}^{\prime}}\right)= \\
& =\mu t\left(1+\frac{3}{8}\left(1+\frac{1}{1-2\left(k^{\prime}\right)^{2}}\right)\left(\frac{\pi / k \mathbf{K}^{\prime}}{\operatorname{ch}\left(\pi \mathbf{K} / 2 \mathbf{K}^{\prime}\right)}\right)^{2}-\frac{\pi}{2 \mathbf{K}^{\prime}} \frac{1}{\sqrt{1-2\left(k^{\prime}\right)^{2}}}\right) .
\end{aligned}
$$

Оценим вклады, связанные с разностью аргументов. Имеем

$$
s_{0}(t)-s_{0}^{(r)}(t)=A \operatorname{sh}(\mu t \boldsymbol{\Omega})\left(1-\operatorname{ch} \Delta_{r}\right)-A \operatorname{ch}(\mu t \boldsymbol{\Omega}) \operatorname{sh} \Delta_{r} .
$$

При малых $\Delta$, например при $|\Delta|<1$, имеют место неравенства

$$
|\operatorname{sh} \Delta|<2|\Delta|, \quad|1-\operatorname{ch} \Delta|<2|\Delta|,
$$

отсюда при $t>0$ и $\Delta_{r}<1$ получаем оценку

$$
\left|s_{0}(t)-s_{0}^{(r)}(t)\right|<2\left|\Delta_{r}\right| A \operatorname{sh}(\mu t \boldsymbol{\Omega})+2\left|\Delta_{r}\right| A \operatorname{ch}(\mu t \boldsymbol{\Omega})=2\left|\Delta_{r}\right| A e^{\mu t \boldsymbol{\Omega}} .
$$

Видно, что точность приближения $s_{0}^{(r)}(t)$ к $s_{0}(t)$ определяется малостью $\left|\Delta_{r}\right|$.

Заметим, что аналогичное утверждение верно и для приближения, содержащего $n$ мод: для $\left|\Delta_{r}\right|<1$

$$
\left|s_{n}(t)-s_{n}^{(\text {exact }, r)}(t)\right|<2\left|\Delta_{r}\right| A \operatorname{sh}(\mu t \boldsymbol{\Omega})+2\left|\Delta_{r}\right| A_{n} \operatorname{ch}(\mu t \boldsymbol{\Omega})=2\left|\Delta_{r}\right| A_{n} e^{\mu \boldsymbol{\Omega} t} .
$$

Из явных выражений для $\delta_{0}$ и $\delta_{1}$ вытекает, что $\left|\delta_{0}\right|<\sqrt{2}\left(k^{\prime}\right)^{2}$ и $\delta_{1}<\left(k^{\prime}\right)^{4}$ для $0 \leqslant k^{\prime}<1 / 2$, следовательно,

$$
\left|\Delta_{0}\right|<\sqrt{2} \mu t\left(k^{\prime}\right)^{2}, \quad \Delta_{1}<\mu t\left(k^{\prime}\right)^{4} .
$$


5.3.4. Приближение, содержащее две моды. Рассмотрим приближение $q_{1}(t)=$ $q_{0}(t)+s_{1}(t)$, определенное формулами (5.2)-(5.4), и в качестве приближения к $s_{1}(t)$ возьмем

или

$$
\bar{s}_{1}^{(0,0)}=-\frac{A^{3} \epsilon}{32 \mu^{2}} \operatorname{sh}(3 \mu t)
$$

$$
s_{1}^{(0,0)}(t)=-A\left(k^{\prime}\right)^{2} \frac{1}{16} \operatorname{sh}(3 \mu t) .
$$

Тогда отклонение $\bar{s}_{1}^{(0,0)}$ от $s_{1}(t)$ связано не только с изменением аргумента гиперболического синуса, которое равно $3 \Delta_{0}$, но и с поправками к точному значению $A_{1}=\left(k^{\prime}\right)^{2} A / 16+a\left(k^{\prime}\right)^{5} L_{1}\left(k^{\prime}\right)$. В силу неравенства

$$
-\frac{\left(k^{\prime}\right)^{2}}{16} \frac{\pi}{k \mathbf{K}^{\prime}} \frac{1}{\operatorname{ch}\left(\pi \mathbf{K} / 2 \mathbf{K}^{\prime}\right)}+\frac{\pi}{k \mathbf{K}^{\prime}} \frac{1}{\operatorname{ch}\left(3 \pi \mathbf{K} / 2 \mathbf{K}^{\prime}\right)}<\frac{1}{10}\left(k^{\prime}\right)^{5},
$$

справедливого при $0 \leqslant k^{\prime}<1 / 2$, имеет место оценка $L_{1}\left(k^{\prime}\right)<1 / 10$.

Таким образом,

$$
\begin{aligned}
& s_{1}^{(0,0)}(t)-s_{1}(t)=-A \frac{\left(k^{\prime}\right)^{2}}{16} \operatorname{sh}(3 \mu t)+A_{1} \operatorname{sh}(3 \boldsymbol{\Omega} t)= \\
& \quad=-A \frac{\left(k^{\prime}\right)^{2}}{16}\left(\operatorname{sh}\left(3 \mu t\left(\delta_{0}+\boldsymbol{\Omega}\right)\right)-\operatorname{sh}(3 \mu t \boldsymbol{\Omega})\right)+\left(A \frac{\left(k^{\prime}\right)^{2}}{16}+A_{1}\right) \operatorname{sh}(3 \mu t \boldsymbol{\Omega}) .
\end{aligned}
$$

С учетом оценок типа (5.12) и (5.17) окончательно получаем

$$
\left|s_{1}^{(0,0)}(t)-s_{1}(t)\right|<C\left(k^{\prime}\right)^{4} e^{3 \mu t \Omega}, \quad C=\text { const } .
$$

5.3.5. Явная оценка точности приближения второго порядка по $\epsilon$ для модифицированной теории возмущений. Имеет место следующая

ТЕОРема 2. Пусть $0<c<1 / 2$. Тогда для любого $\epsilon>0$ найдутся постоянные $C>0$ и $\sigma<3 / 4$ такие, что при всех

$$
0 \leqslant t \leqslant \frac{c}{2 \sqrt{2} \mu} \ln \frac{\mu^{4}}{2 \epsilon E}
$$

выполнена ощенка

$$
\left|q(t)-s_{0}^{(1)}(t)-s_{1}^{(0,0)}(t)\right| \leqslant C \frac{\sqrt{E}}{\mu}\left(\frac{\epsilon E}{\mu^{4}}\right)^{2-\sigma} .
$$

ЗАмЕчаниЕ 2. В оценке (5.19) $C$ - безразмерная постоянная, множитель $\sqrt{E} / \mu$ написан из соображений размерности, $\epsilon E / \mu^{4}-$ безразмерный множитель.

ДокАЗАТЕЛЬство. Согласно (5.4) $q_{1}(t)=q_{0}(t)+s_{1}(t)$. В оценке (5.19) мы взяли приближение $s_{0}^{(1)}(t)$ к $q_{0}(t)$ и приближение $s_{1}^{(0,0)}$ к $s_{1}(t)$. Мы имеем

$$
q(t)-s_{0}^{(1)}(t)-s_{1}^{(0,0)}=q(t)-q_{1}(t)+q_{0}(t)-s_{0}^{(1)}(t)+s_{1}(t)-s_{1}^{(0,0)}(t),
$$

следовательно,

$$
\left|q(t)-s_{0}^{(1)}(t)-s_{1}^{(0,0)}\right|<\left|q(t)-q_{1}(t)\right|+\left|q_{0}(t)-s_{0}^{(1)}(t)\right|+\left|s_{1}(t)-s_{1}^{(0,0)}(t)\right| .
$$

Первое слагаемое в правой части данного неравенства при выполнении условия (5.18) удовлетворяет оценке из теоремы 1. Для завершения доказательства используются оценки (5.13) и (5.14). 
Благодарности. Авторы благодарны В. В. Козлову и Д. В. Трещёву за полезные обсуждения. Работа была частично поддержана РФФИ (гранты № 11-01-00894_а (И. Арефьева), 11-01-00828_а (И. Волович и Е. Писковский) и 11-01-12114-офи_м (И. Волович)), а также Программой поддержки ведущих научных школ (гранты НШ-4612.2012.1 (И. Арефьева), НШ-2928.2012.1 (И. Волович и Е. Писковский)).

\section{Список литературы}

[1] Н. М. Крылов, Н. Н. Боголюбов, Введение в нелинейную механику, Изд-во АН УССР, Киев, 1937.

[2] Н.Н. Боголюбов, Ю.А. Митропольский, Асимптотические методы в теории нелинейных колебаний, Наука, М., 2005.

[3] В. И. Арнольд, В. В. Козлов, А. И. Нейштадт, Математические аспекты классической и небесной механики, ВИНИТИ, М., 1985.

[4] В. В. Козлов, С. Д. Фурта, Асимптотики решений сильно нелинейных систем дифберенииальных уравнений, НИЦ "Регулярная и хаотическая динамика", Ижевск, 2009.

[5] В. А. Рубаков, Классические калибровочные поля, УРСС, М., 1999.

[6] V.F. Mukhanov, Physical Foundations of Cosmology, Cambridge Univ. Press, Cambridge, 2005.

[7] Д. С. Горбунов, В. А. Рубаков, Введение в теорию ранней Вселенной. Космологические возмущения. Инфляиионная теория, ИЯИ РАН, М., 2009.

[8] I. Ya. Aref'eva, I. V. Volovich, JHEP, 8 (2011), 102, 32 pp., arXiv: 1103.0273.

[9] I. Ya. Aref'eva, N. V. Bulatov, R. V. Gorbachev, FRW cosmology with non-positively defined Higgs potentials, arXiv: 1112.5951.

[10] И. В. Волович, ТМФ, 164:3 (2010), 354-362.

[11] E. V. Piskovskiy, I. V. Volovich, "On the correspondence between Newtonian and functional mechanics", Quantum Bio-Informatics IV: From Quantum Information to Bio-informatics (Tokyo, Japan, March 10-13, 2010), Quantum Probability and White Noise Analysis, 28, eds. L. Accardi, W. Freudenberg, M. Ohaya, World Scientific, Hackensack, NJ, 2011, 363-372.

[12] А. М. ЖХравский, Справочник по эллиптическим функииям, Наука, М., 1941.

[13] Н. И. Ахиезер, Элементы теории эллиптических функиий, М., Наука, 1970.

[14] М. Абрамовиц, И. Стиган, Справочник по специальным функииям с формулами, графиками и математическими таблицами, Наука, М., 1979.

Поступила в редакцию 9.02.2012 